\title{
Positive solutions to semilinear problems with coefficient that changes sign
}

\author{
Nguyen Phuong Cac, Juan A. Gatica, Yi Li \\ Department of Mathematics, University of Iowa; \\ Iowa City IA 52242, USA
}

\section{Introduction}

In this paper we will discuss the existence'and non-existence of positive solutions to the problem:

$$
\begin{aligned}
& -\Delta u=\lambda a(x) f(u), x \in \Omega \\
& u(x)=0, x \in \partial \Omega .
\end{aligned}
$$

where $\Omega$ will be assumed to be a bounded domain in $R^{N}$ with smooth boundary.

We will concentrate on the case when the coefficient $a(x)$ is allowed to change sign; this brings about an interesting mathematical problem.

This is not a new problem: due to its appearance in many mathematical models in physics, several special cases of it have been studied since the middle 1800's. For example, it was used by Lord Kelvin $[\mathrm{T}, \mathrm{W}]$ and J. Lane $[\mathrm{L}, \mathrm{J}]$ to study the equilibrium configuration of mass in a spherical cloud gas; this model was further studied by R.H. Fowler $[\mathrm{F}, \mathrm{R}]$, leaving the Emden Fowler equation which, in a generalized form is still studied today, as seen in, for example [AP]. For a review of the work done up to 1975 in the ordinary differential equations setting, see [W]; for a review of the work done for the existence of positive solutions of semilinear elliptic equations, see [L].

Positive solutions are usually the ones of interest and because of the difficulties that this brings in using the techniques developed for nonlinear functional analysis, most of the recent work assumes nonnegativity of $a(x)$ and $f(u)$ in order to generate positive operators using the Green's function of $-\Delta$ on the region where the problem is posed.

In nuclear physics, a form of (1.1) was proposed and used by E. Fermi [F,E] and by L. H. Thomas $[\mathrm{T}, \mathrm{L}]$, and now it is widely known as the Thomas-Fermi equation.

Both the Emden-Fowler equation as well as the Thomas Fermi equation continue to be subjects of considerable interest in theoretical physics to this day, as a search on the Wide World Web will quickly convince you.

In combustion theory equation (1.1) has also been used as a model, for example in $[\mathrm{ACP}],[\mathrm{BK}],[\mathrm{CL}],[\mathrm{G}],[\mathrm{L}]$.

Equation (1.1) has also found applications to geometry: see [N]

As mentioned above, most of the literature deals with the problem under the assumption that the coefficient function $a(x)$ be positive in its domain; we are interested in the case when this function is allowed to change sign. In particular we are interested in determining sufficient conditions on $a(x)$ to assure the existence of positive solutions for 
small values of $\lambda$, to determine ranges of $\lambda$ for which no positive solution exists, and to determine conditions under which there are more than one positive solution.

The main motivation for this work are the inherent mathematical difficulties it presents.

We consider the problem of establishing conditions for the existence of positive solutions of equation (1.1).

\section{The general case: existence.}

In this section we will tackle the general case of existence of positive solutions of (1.1), imposing as few conditions on $\Omega$ as we can and, in particular, we will consider both the radially symmetric as well as the non-radially symmetric cases.

In another section we will also study the case when positive solutions can be ruled out for values of $\lambda$ in certain ranges.

Theorem 2.1. Suppose that $f \in L^{\infty}(R) ; a \in L^{s}(\Omega), s>1$. Then for every $\lambda \in R$ problem (1.1) has a solution $u_{\lambda} \in W^{2, s}(\Omega)$.

Proof.

For any $v \in L^{s}(\Omega)$ and any $\mu \in[0,1]$ let $T_{\mu}(v) \in W^{2, s}(\Omega)$ be the solution to the Dirichlet problem

$$
\begin{aligned}
& -\Delta u=\mu \lambda a(x) f(v(x)), x \in \Omega \\
& u(x)=0, x \in \partial \Omega .
\end{aligned}
$$

For some constant $K>0$, independent of $v, \mu, \lambda$, we have:

$$
\begin{array}{r}
\left\|T_{\mu}(v)\right\| \leq\left\|T_{\mu}(v)\right\|_{W^{2, s}} \leq K|\lambda|\|a\|_{L^{s}}=\operatorname{deg}\left(I-T_{0}, B_{\rho}(0), 0\right) \\
=\operatorname{deg}\left(I, B_{\rho}(0), 0\right)=1, \quad 0 \leq \mu \leq 1 .
\end{array}
$$

Thus $T_{1}$ has a fixed point inside $B_{\rho}(0)$ and this implies that the Dirichlet problem (2.1) has a solution $u \in W^{2, s}(\Omega)$.

Theorem 2.2. Let $a \in L^{s}(\Omega), s>N / 2$, and suppose that there exists $\varepsilon>0$ such that the solution of the Dirichlet problem

$$
\begin{aligned}
& -\Delta w=a^{+}(x)-(1+\varepsilon) a^{-}(x), x \in \Omega \\
& w(x)=0, x \in \partial \Omega,
\end{aligned}
$$

where $a^{+}(x)=\max (0, a(x)), a^{-}(x)=a^{+}(x)-a(x), x \in \Omega$ is nonnegative inside $\Omega$. Suppose further that $f \in C(R, R), f(0)>0$. Then there exists $\lambda_{0}>0$ such that for $\lambda \in\left(0, \lambda_{0}\right)$ the Dirichlet problem

$$
\begin{aligned}
-\Delta u & =\lambda a(x) f(u), x \in \Omega \\
u & =0 \text { on } \partial \Omega
\end{aligned}
$$

has a solution which is positive in $\Omega$.

Proof. 
Fix a large number $M>0$ and define $g: R \rightarrow R$ as follows:

$$
\begin{aligned}
g(\zeta) & =f(0), \zeta \leq 0, \\
g(\zeta) & =f(\zeta), 0<\zeta \leq M \\
g(\zeta) & =f(M), M<\zeta .
\end{aligned}
$$

By Theorem 1, the Dirichlet problem

$$
\begin{aligned}
-\Delta u & =\lambda a(x) g(u), x \in \Omega, \\
u & =0 \text { on } \partial \Omega
\end{aligned}
$$

has a solution $u_{\lambda} \in W^{2, s}(\Omega)$. Since $s>/ 2$, by Sobolev's imbedding theorem, implies that $u \in C(\bar{\Omega})$. that

Fix a number $\gamma \in\left(0, \frac{\varepsilon}{2+\varepsilon}\right)$. The continuity of $g$ implies that there is $\delta \in(0, M)$ such

$$
|\zeta|<\delta \Rightarrow g(0)-g(0) \gamma<g(\zeta)<g(0)+g(0) \gamma
$$

Again the fact that $s>N / 2,(2.2)$ and the Sobolev imbedding theorem imply that there exists $\lambda_{0}>0$ such that

$$
\lambda \in\left(0, \lambda_{0}\right) \Rightarrow\left\|u_{\lambda}\right\|_{C(\bar{\Omega})}<\delta .
$$

If we denote by $G: \Omega \times \Omega \rightarrow R$ the Green's function for $-\Delta$ with homogeneous boundary conditions, then it is well known that $G(x, \zeta)>0,(x, \zeta) \in \Omega \times \Omega$, and, for $x \in \Omega$ and $\lambda \in\left(0, \lambda_{0}\right)$ :

$$
\begin{aligned}
u_{\lambda}(x) & =\lambda\left\{\int_{\Omega} G(x, \zeta) a^{+}(\zeta) g\left(u_{\lambda}(\zeta)\right) d \zeta-\int_{\Omega} G(x, \zeta) a^{-}(\zeta) g\left(u_{\lambda}(\zeta)\right) d \zeta\right\} \\
& >\lambda \int_{\Omega} G(x, \zeta)\left\{a^{+}(\zeta)[g(0)-g(0) \gamma]-a^{-}(\zeta)[g(0)+g(0) \gamma]\right\} d \zeta \\
& >\lambda f(0)(1-\gamma) \int_{\Omega} G(x, \zeta)\left[a^{+}(\zeta)-\frac{1+\gamma}{1-\gamma} a^{-}(\zeta)\right] d \zeta
\end{aligned}
$$

From the hypothesis concerning problem (2.3), it follows that:

$$
u_{\lambda}(x)>\lambda f(0)(1-\gamma)\left[(1+\varepsilon)-\frac{1+\gamma}{1-\gamma}\right] \int_{\Omega} G(x, \zeta) a^{-}(\zeta) d \zeta
$$
$\Omega$.

By our choice of $\gamma$ the right hand side is nonnegative and thus we have $u_{\lambda}(x)>0, x \in$

Remark 2.3. Theorem 2 improves the result of $[\mathrm{CFG}]$ in a number of ways.

First: [CFG] considers only the radially symmetric case.

Second: it uses the method of upper and lower solutions and, as a consequence of the method, it requires that $f$ be nondecreasing.

Remark 2.4. Third: [CFG] requires that for some $\tau>0$ 


$$
\int_{0}^{t} r^{N-1} a^{+}(r) d r \geq(1+\tau) \int_{0}^{t} r^{N-1} a^{-}(r) d r, 0 \leq t \leq \nu
$$

where $\nu$ is the radius of the ball $\Omega=B_{\nu}(0)$.

This condition implies that the radially symmetric solution $u(x)=u(\|x\|)$ of $(1.1)$ is positive inside the ball $\Omega$. In fact, since $a$ cannot be zero in $\Omega$, if $a^{-}(r)=0,0 \leq r \leq \nu$ then, on the one hand, (2.4) holds for any $\tau>0$; on the other hand for any $\varepsilon>0$ the solution of (2.3) is positive inside the ball by the maximum principle. Hence Theorem 2.2 generalizes the main result in $[\mathrm{CFG}\}$

So let us consider the case $a^{-}$not identically zero on $[0, \nu]$. Let $\omega(x)=\omega(\|x\|)$ satisfy:

$$
\begin{aligned}
& -\Delta \omega=a^{+}(x)-(1+\tau / 2) a^{-}(x),\|x\|<v \\
& \omega(x)=0,\|x\|=v .
\end{aligned}
$$

Then:

$$
\begin{aligned}
& {\left[\omega(r) r^{N-1}\right]^{\prime}=-r^{N-1}\left[a^{+}(r)-(1+\tau / 2) a^{-}(r)\right], 0 \leq r \leq v} \\
& \omega(\gamma)=0, \omega^{\prime}(0)=0
\end{aligned}
$$

Thus:

$$
\begin{aligned}
& \omega^{\prime}(r) r^{N-1}=-\int_{0}^{r} t^{N-1} a^{+}(t) d t+(1+\tau / 2) \int_{0}^{r} t^{N-1} a^{-}(t) d t \\
& \leq-(\tau / 2) \int_{0}^{r} t^{N-1} a^{-}(t) d t, 0 \leq r \leq v
\end{aligned}
$$

Since $a^{-}$is not identically zero, there exists $r_{0} \in(0, \nu)$ such that the right hand side of 1.2.7 is negative on $\left[r_{0}, \nu\right]$. It follows that $\omega^{\prime}$ is nonpositive on $[0, \nu]$ and negative on $\left[r_{0}, \nu\right]$. Since $\omega(\nu)=0$ it follows that the solution $\omega$ is positive inside the ball. Thus hypothesis (4) of $[\mathrm{CFG}]$ is stronger than our hypothesis concerning equation (1.1).

We end this section with a result that holds in our general setting and will be of considerable importance in what is to come.

Corollary 2.5. If $\Omega$ is a ball, $f \in C(\Omega), f(0)>0, a \in L^{s}(\Omega), \dot{s}>N / 2$, and if the problem is radially symmetric, then there exists $\lambda_{0} \in(0,1)$ such that problem (2.1) has a positive solution for $\lambda \in\left(0, \lambda_{0}\right)$.

Lemma 2.1. Let $a \in L^{s}(\Omega), s>N / 2, a \neq 0$, and consider the problem:

$$
\begin{aligned}
& -\Delta \omega=a(x), x \in \Omega \\
& -\Delta \omega_{1}=a(x) \omega, x \in \Omega \\
& \omega=\omega_{1}=0 \text { on } \partial \Omega .
\end{aligned}
$$

If $\partial \Omega$ satisfies Hopf's boundary lemma, then

$$
\frac{\partial \omega_{1}}{\partial v}<0
$$

on $\partial \Omega$, where $v$ is the unit outward normal vector.

Proof.

Let $W(x)=\omega_{1}(x)-\frac{1}{2} \omega^{2}(x)$. Then:

$$
\begin{aligned}
\Delta W(x) & =\Delta \omega_{1}(x)-\left[\omega(x) \Delta \omega(x)+|\nabla \omega|^{2}(x)\right] \\
& =-|\nabla \omega|^{2}(x) .
\end{aligned}
$$


It follows that $W$ is a strict super harmonic function on $\Omega$ unless $\nabla \omega$ is identically zero, which would imply that $\omega$ is identically zero and thus a must be identically zero.

Thus, under our assumptions, it must be the case that $\Delta W(x) \leq 0$ and is not identically zero on $\partial \Omega$.

Hopf's boundary lemma now yields:

$$
0>\frac{\partial W}{\partial v}=\frac{\partial \omega_{1}}{\partial v}-\omega \frac{\partial \omega}{\partial v}=\frac{\partial \omega_{1}}{\partial v} .
$$

\section{$3 \quad$ Existence and Non-Existence.}

Now we will concentrate our focus in adding conditions which will assure us of much more detailed information concerning the existence or non-existence of positive solutions for the problem under study.

The most used hypotheses in this section are listed below. In specific instances we will add to them.

$\left.H_{1}\right) f: R \rightarrow R$ is continuous with $f(0)>0$.

$\left.H_{2}\right) a \in L^{s}(\Omega), \dot{s}>N / 2$.

$\left.H_{3}\right) \Omega$ is a bounded domain in $R^{N}, 0 \in \Omega$, and $\partial \Omega \in C^{2}(\Omega)$.

$\left.H_{4}\right)_{\varepsilon} \int_{\Omega} G(x, \zeta)\left(a^{+}(\zeta)-(1+\varepsilon) a^{-}(\zeta)\right) d \zeta>0, x \in \Omega$.

$\left.H_{5}\right) A(t)=\int_{0}^{t} r^{N-1} a(r) d r \geq 0$ and for some $t_{0} \in(0,1), A\left(t_{0}\right)>0$.

A direct consequence of Theorem 2 is the following:

Theorem 3.1. Under $\left.H_{1}\right)$ and $\left.H_{4}\right)_{\varepsilon}$ for some $\varepsilon>0$, there exists $\lambda_{0} \in(0,1)$ such that problem (1.1) has at least one positive solution for $\lambda \in\left(0, \lambda_{0}\right)$.

Now we will prove our first nonexistence result.

Theorem 3.2. If $\left.\left.H_{2}\right), H_{4}\right)_{0}$ hold, if $B_{1}(0) \subseteq \Omega$ and if $f^{\prime}(0)<0$ then (1.1) has no bounded positive in solution in $L^{\infty}$ for $\lambda>0$.

Proof.

Let $\rho>0$ and define

$$
\begin{aligned}
b(r) & =1,0 \leq r \leq \rho \\
& =-1, \rho \leq r \leq 1 .
\end{aligned}
$$

Define

$$
\omega(x)=\int_{B_{1}(0)} G(x, \zeta) b(\zeta) d \zeta
$$

where $G$ is the Green's function for $-\Delta$ on $B_{1}(0)$.

Given our assumptions, there exists $\rho>0$ so that:

$$
\begin{aligned}
\omega(x) & >0, x \in B_{1}(0), \\
\frac{\partial \omega}{\partial v} & =0 \text { on } \partial B_{1}(0) .
\end{aligned}
$$

Let $u=\lambda W$ be any solution of (1.1). Then:

$$
-\Delta W(x)=a(x) f(\lambda w(x)) .
$$


We have:

$$
\begin{aligned}
W(x) & =\int_{B_{1}(0)} G(x, \zeta) a(\zeta) f(\lambda W(\zeta)) d \zeta \\
& =\int_{B_{1}(0)} G(x, \zeta) a(\zeta)\left[f(0)+f^{\prime}(0) \lambda W(\zeta)+o(W(\zeta))\right] d \zeta \\
& =f(0) \omega(x)+\lambda f^{\prime}(0) \int_{B_{1}(0)} G(x, \zeta) a(\zeta) W(\zeta)[1+o(1)] d \zeta \\
& =f(0) \omega(x)+\lambda f^{\prime}(0) \omega_{\lambda}(x),
\end{aligned}
$$

where

$$
\omega_{\lambda}(x)=\int_{B_{1}(0)} G(x, \zeta) a(\zeta) W(\zeta)[1+o(1)] d \zeta .
$$

As $\lambda \rightarrow 0^{+},(3.1)$ becomes $\Delta W_{0}+a f(0) \geq 0$, concluding that $W_{0}=f(0) \omega(x)$ and hence

$$
\omega_{\lambda}(x) \rightarrow \int_{B_{1}(0)} G(x \zeta) a(\zeta) \omega(\zeta) f(0) d \zeta=\omega_{2}(x) .
$$

Here we have

$$
\begin{aligned}
-\Delta \omega_{2}(x) & =f(0) a(x) \omega(x) \text { on } B_{1}(0), \\
\frac{\partial \omega_{2}}{\partial v} & <0 \text { on } \partial B_{1}(0) .
\end{aligned}
$$

Hence

$$
\frac{\partial \omega_{\lambda}}{\partial v}<0
$$

for $\lambda$ sufficiently small. For these values of $\lambda$ we get:

$$
\frac{\partial W}{\partial v}=f(0) \frac{\partial \omega}{\partial v}+\lambda f^{\prime}(0) \frac{\partial \omega_{\lambda}}{\partial v}>0
$$

and this together with the fact that $W=0$ on $\partial B_{1}(0)$ implies that $W$ is negative near the boundary.

Remark 3.3. The same argument implies that if $f^{\prime}(0)>0$ and $\frac{\partial \omega_{2}}{\partial v}<0$ on $\partial B_{1}(0)$ then $W$ is positive for $\lambda$ small and positive.

An immediate consequence is:

Theorem 3.4. If $\left.H_{2}\right)$ and $\left.H_{4}\right)_{0}$ hold, if $\Omega=B_{1}(0)$ and if $f^{\prime}(0)>0$ then there is $\lambda_{0} \in(0,1)$ such that problem (1.1) has a positive solution for $\lambda \in\left(0, \lambda_{0}\right)$.

Theorem 3.5. Assume that the $\Omega=B_{1}(0)$, that the problem is radially symmetric, that $a:[0,1] \rightarrow R$ is nonincreasing, that $f: R \rightarrow R$ is locally Lipschitz and positive and such that there exists a positive nondecreasing function $g$ with $g(u)=\min _{s \geq u} f(s)$ satisfies the strictly superlinear growth condition $\int_{0}^{\infty} \frac{d u}{g(u)}<\infty$ and there exists $\varepsilon>0$ such that $a(x)>\varepsilon$ in $B_{\varepsilon}(0)$.

Then the problem

$$
\begin{aligned}
-\Delta u & =\lambda a f(u) \text { in } B_{1}(0), \\
u & >0 \text { in } B_{1}(0) \\
u & =0 \text { on } \partial B_{1}(0)
\end{aligned}
$$

has no solution if $\lambda \geq \frac{2 N}{\varepsilon^{3}} \int_{0}^{\infty} \frac{d u}{g(u)}$. 
Proof.

If $u$ is a positive solution, then by [GNN], $u$ is radially symmetric and so:

$$
u^{\prime \prime}+\frac{N-1}{r} u^{\prime}+\lambda a(r) f(u)=0 .
$$

In $B_{\varepsilon}(0)$ we then have:

$$
r^{N-1} u^{\prime}(r)+\lambda \int_{0}^{r} s^{N-1} a(s) f(u(s)) d s=0 .
$$

This implies that

$$
r^{N-1} u^{\prime}(r)+\lambda \varepsilon \int_{0}^{r} s^{N-1} g(u(s) d s \leq 0 .
$$

Since $u^{\prime}<0$ in $(0, \varepsilon)$, we have that:

$$
r^{N-1} u^{\prime}(r)+\lambda \varepsilon g(u(r)) \int_{0}^{r} s^{N-1} d s \leq 0 .
$$

We get:

$$
\frac{u^{\prime}(r)}{g(u)}+\frac{\lambda \varepsilon}{N} r \leq 0
$$

Thus:

$$
\int_{0}^{\varepsilon} \frac{u^{\prime}(r)}{g(u(r))} d r+\int_{0}^{\varepsilon} \frac{\lambda \varepsilon}{N} r d r \leq 0
$$

This shows that:

$$
\frac{\lambda \varepsilon^{3}}{2 N} \leq \int_{u(\varepsilon)}^{u(0)} \frac{d u}{g(u)} \leq \int_{0}^{\infty} \frac{d u}{g(u)} .
$$

This yields the desired result.

Theorem 3.6. If there exists $\varepsilon>0$ such that $B_{\varepsilon}\left(x_{0}\right) \subseteq \Omega$, if $a(x) \geq \varepsilon$ on $B_{\varepsilon}\left(x_{0}\right)$ and if $f(u) \geq g(u) \geq 0$, where $g$ is nondecreasing, convex, with $\int_{0}^{\infty} \frac{d u}{g(u)}<\infty$, then the problem

$$
\begin{aligned}
-\Delta u & =\lambda a(x) f(u) \text { in } B_{\varepsilon}\left(x_{0}\right) \\
u & >0 \text { in } B_{\varepsilon}\left(x_{0}\right)
\end{aligned}
$$

has no solution if

$$
\lambda \geq \frac{2 N}{\varepsilon^{4}} \int_{0}^{\infty} \frac{d u}{g(u)} .
$$

Proof.

Let $\bar{u}(r)=\int_{\partial B_{r}\left(x_{0}\right)} u(x) d S_{x}$ for $0<r<\varepsilon$.

In $B_{\varepsilon}\left(x_{0}\right)$ we have:

$$
\int_{\partial B_{r}\left(x_{0}\right)}[\Delta u+a f(u)]=0
$$

and hence:

$$
\int_{B_{r}\left(x_{0}\right)}[\Delta u+\lambda \varepsilon g(u)] \leq 0
$$

in $B_{\varepsilon}\left(x_{0}\right)$. 
Jensen's inequality implies that:

$$
\Delta \bar{u}+\lambda \varepsilon^{2} g(u) \leq 0
$$

Similarly we get:

$$
\lambda<\frac{2 N}{\varepsilon^{4}} \int_{0}^{\infty} \frac{d u}{g(u)}
$$

\section{References.}

[ACP] D. Aronson, M. G. Crandall, and L. A. Peletier, Stabilization of solutions of a degenerate nonlinear diffusion problem, Nonlinear Analysis, T.M.A., 6 (1982), 1001-1022.

[AP] F. V. Atkinson and L. A. Peletier, Ground states of $-\Delta u=f(u)$ and the EmdenFowler Equation, Arch. Rat. Mech. Anal., 93 (1986), 103-127.

[BK] J. W. Bebernes and D. R. Kassoy, A mathematical analysis of blowup for thermal reactions - the spatially inhomogeneous case, SIAM J. App. Math. 40, (1981),476-484.

[CFG] N. P. Cac, A. M. Fink and J. A. Gatica, Nonegative solutions to the radial Laplacian with nonlinearity that changes sign, Proc. A. M. S., 123 (1995),1393-138.

[CL] Y. S. Choi and G. S. S. Ludford, An unexpected stability result of near-extinction flame of non-unity Lewis numbers, Quart. J. Mech. Appl. Math., 42 (1989), 143-158.

[DL] Y. Deng and Y. Li, Existence and bifurcation of the positive solutions for a semilinear equation with critical exponent, J. D. E. 130, (1996), 179-200.

[G] I. M. Gelfand, Some problems in the theory of quasilinear equations, Uspekhi Mat. Nauka, 14 (1963), 295-381.

$[\mathrm{F}, \mathrm{E}]$, E. Fermi, Un metodo stadistico per la determinazione di alcune proprieta dell atome, Rend. Acad. Naz. del Lincei, CL, Sci. Fis., Mat. e Nat., 6 (1927), 602-607.

$[F, R]$ R. H. Fowler, Further studies of Emden's and similar differential equations, Quar. J. Math. 2 (1931), 259-288.

[GNN] B. Gidas, W.-M. Ni, L. Niremberg, Symmetry of positive solutions of nonlinear elliptic equations in $R^{n}$, Advances in Math., Supplementary Studies, 7A (1981),369-402.

[KLO] Phillip Korman, Yi Li and Tianchen Ouyang, Exact multiplicity results for boundary value problems generalizing cubic, Proc. Royal Soc. Edinburgh, 126A (1996), 599-616.

[L] P. L. Lions, On the existence of positive solutions of semilinear elliptic equations, SIAM Review, 24 (1882), 441-467.

$[\mathrm{L}, \mathrm{J}]$ L. J. Homer Lane, On the theoretical temperature of the sun under the hypothesis of a gaseous mass maintaining its volume by its internal heat and depending on the laws of gases known to terrestial experiment, Amer. J. Sci. and Arts,4 (1869-70),57-74.

[LN] Yi Li and Wei-Ming Ni, On the existence and symmetry properties of finite total mass solutions of the Matukama equation, the Eddington equation and their generalizations, Arch. Rat. Mech. Anal., 108 (1989), 175-194.

[N] Wei-Ming Ni, On the elliptic equation $\Delta u+K(x) e^{2 u}=0$ and conformal metrics with prescribed Gaussian curvatures.

$[$ T,L] L. H. Thomas, The calculation of atomic fields, Proc. Camb. Phil. Soc., 23 (1927), 542-548.

$[\mathrm{T}, \mathrm{W}]$ Thompson, W. (Lord Kelvin), On the convective equilibrium of temperature in atmosphere, Manchester Philos. Soc. Proc. 2 (1860-62),170-176, reprint. Math. and Phys. Papers by Lord Kelvin, 3 (1890), 255-60. 
[W] Wong, J. S. W., On the generalized Emden-Fowler equation, SIAM Review, 17 (1975), 339-360. 